\title{
Design status of the ITER ECH Upper Launcher Steering Mirror Mechanism
}

\author{
J.-D. Landis ${ }^{\mathrm{a}}$, R. Chavan ${ }^{\mathrm{a}}$, M. Henderson ${ }^{\mathrm{a}}$, R. Bertizzolo ${ }^{\mathrm{a}}$, A. Collazos ${ }^{\mathrm{a}}$, F. Sanchez ${ }^{\mathrm{a}}$ \\ ${ }^{a}$ CRPP - EPFL, Association Euratom Confédération Suisse, 1015 Lausanne, Switzerland
}

\begin{abstract}
The ITER ECRH upper port antenna (or launcher) will be used to drive current locally for stabilising the neoclassical tearing mode (NTM) by depositing mm-wave power inside of the island which forms on the $q=3 / 2$ or 2 rational magnetic flux surfaces and control the sawtooth instability by driving current near the $q=1$ surface. This requires the launcher to be capable of steering the focused beam deposition location across the resonant flux surface over the range where the $q=1,3 / 2$ and 2 surfaces are expected to be found (roughly the outer half of the plasma) [4].
\end{abstract}

ITER's present reference design uses a front steering (FS) concept, which uses a moveable mirror close to the plasma. Two separate mirrors are used to decouple the focusing and steering aspects resulting in an optimized optical configuration providing a well focused beam over a large steering range [6].

The steering mechanism providing the mirror rotation uses a frictionless and backlash free mechanical system based on the elastically compliant deformation of structural components to avoid the in vessel tribological difficulties. Traditional designs are based on push-pull rods acting on a mirror which rotates with ball bearings, they present the risk of gripping or result in stickslip movements. The ball bearings are replaced with a set of flexure pivots while the classic actuation through a push-pull rod scheme is replaced by a directly acting pneumatic system consisting on a fast feed line, bellows and springs, in which the pressure acting on the bellows pushes the mirror against the compressive springs. The rotation of the mirror is thus produced by the counteraction between the forces exerced by the springs and the bellows, themselves piloted by the pressure of the system. A servovalve placed outside of the port plug and connected to the bellows by a small tube will control this pressure. The system also includes flexible water cooling pipes which allow the removal of heat generated by the ohmic surface losses of the reflected $\mathbf{m m}$ wave beams and the nuclear and radiation volumic heating of the rotating mirror components.

This paper will give an overview of the engineering and design issues and their solutions, and provide the development status of the different components of the mechanism. Special attention will be given to the engineering analysis performed to ensure compliance of the steering mechanism with the various ITER requirements.

ITER ECRH upper port antenna; Steering mirror; Bellow; Flexure pivot;

\section{INTRODUCTION}

The purpose of the ITER electron cyclotron resonance heating $(\mathrm{ECRH})$ antennae located in the upper port launcher will be to stabilize the neoclassical tearing modes (NTM) by driving currents locally inside either the $q=3 / 2$ or 2 island. The ITER reference design uses a front steering (FS) concept with the moveable mirror close to the plasma. The FS launcher is capable of steering eight $2 \mathrm{MW}$ mm-wave beams of $170 \mathrm{GHz}$ via two sets of steering mirrors. The launcher is structurally integrated into the upper port plug, itself attached to the upper vacuum vessel port with a flange joint (vacuum seal and mechanical link) and tangential keys (alignment within port). All supply lines such as water cooling, cables and waveguides are contained in the port plug, as connections are to be located behind the plug flange. The four upper ports each contain two steering mechanism assemblies.

In the adverse invessel operating conditions, reliable operation is required to guarantee the availability of the mirror steering mechanism during the 20-year lifetime of ITER. The dynamic performance and steering accuracy of the mirror system are essential for the localized beam power deposition. In order to increase the steering reliability and accuracy, traditional bearing and push-pull rods are avoided, which tend to introduce backlash or grip.

The steering mechanism is the critical component of the FS design and a failure of one mirror steering unit would render four RF beams unusable for NTM stabilization applications. A failed steering mechanism can only be replaced during a normal tokamak opening. Since the rotation angle of the steering mirrors is limited by design to $\pm 7^{\circ}$, there is no risk of beams being reflected backward into the waveguides in case of a malfunction. Critical and non massive components, such as the bellows, are protected from stray radiation by confinement in cavities where mm-waves do not penetrate.

The proposed frictionless and backlash free mechanism design uses elastically compliant structural components to guide and drive the rotating mirror. Frictional contacts are avoided, as traditional ball bearings are replaced with flexure pivots and the movement is controlled using an integrated gaseous helium pneumatic actuator system working against preloaded compressive springs in place of the push pull rods. A pair of coiled cooling pipes with either a single or double wall provides a flexible coolant feed to the mirror.

Resistance to cyclic fatigue is the critical design requirement for mechanically compliant systems. In terms of maximizing reliability, the bellows, the flexure pivots, the return springs and the spiral cooling tubes are the core elements of the mechanism. Prototypes of the critical components are being tested. Corresponding analytical and cyclic fatigue numerical models of the compliant components are to be validated by fatigue bench tests. 


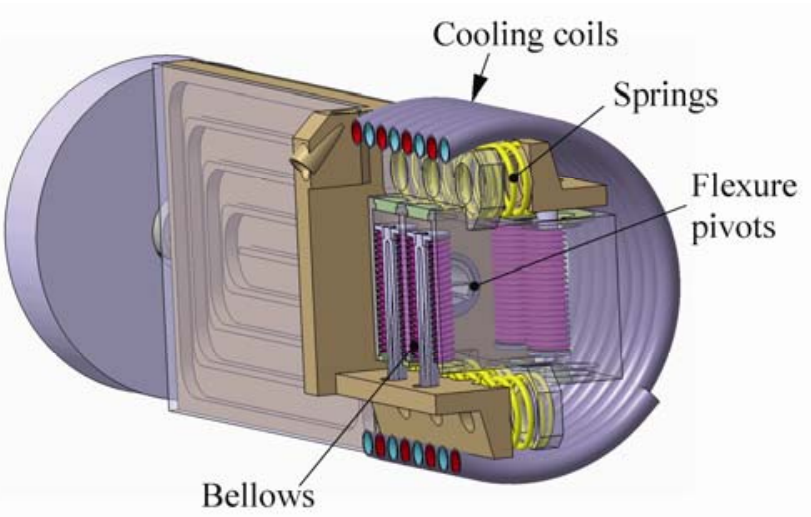

Figure 1. The proposed frictionless and backlash free steering mechanism in its current layout

\section{STEERING MECHANISM COMPONENTS}

A frictionless mechanical system as proposed for the front steering antenna has considerable advantages in the ITER vacuum environment, where beryllium dust contamination, tritium retention, neutron radiation, background vibration and mm-wave arcing are design issues. Tribology related problems are common on all Tokamaks, in particular seizing, cold welding, excessive wear, worsened when relative movements are of small amplitude, in rolling and gliding contacts. Components with gap/play generally require elastic preloading to remove it to avoid vibration or to define position, such as ball bearings, bushings, which adds to the structural loads. Frictionless mechanical links make use of the elastic compliance of parts specifically designed to deform into defined shapes. Relying on flexure and shear deformation, frictionless devices do not require lubrication, do not seize, are backlash free and self-centering. Using materials with low hysteresis, compliant components yield good repeatability. They are simple to install and they are maintenance free during their expected and anticipated lifetime. Failure is due to cyclic fatigue or overloads beyond design values resulting in plastic strain or elastic instability (buckling). Issues related to reliability and failure modes are therefore the main subjects for detailed investigation in the design phase.

The mechanism in its current layout is represented in Fig. 1. As a result of the very tight space restrictions within the port plug front end, the largest possible diameter of the helical cooling coils is imposed, while their cross section is determined by the required quantity of cooling water and the allowed stresses in the pipe walls. Hence, the cooling coils diameter, the number of turns and the length and size of the mirror itself, after being fitted into the front part of the port plug, dictate the volume and distribution of the actuator components within.

Finding the optimal combination of bellows and springs fitting into the steering mirror assembly is an iterative task. The optimization simultaneously takes into account the geometrical, mechanical and fatigue properties of the compliant components, which are the flexure pivots, the bellows, the springs and the cooling coils. The kinematic system contains no intermediary coupling links between the rotating and the fixed parts. The cyclic fatigue may come as a result of the accumulation of local defects related to plastic strain of both large rotations, the neoclassical tearing modes (NTM) switching cycles (associated with rotating the steering mirror between the $\mathrm{q}=3 / 2$ and 2 flux surface) and small rotations, the modulation cycles (small amplitude variations in steering angle in tracking the evolution of the NTM). The magnitude of the NTM switching rotations is estimated to be $5^{\circ}$ or equivalent to $50 \%$ of the total rotation range, based on the average difference in maximum steering angles for the $\mathrm{q}=2$ to $3 / 2$ magnetic surfaces for the ITER scenarios 2, 3a and 5. The magnitude of the modulation rotation should not exceed $2^{\circ}$, based on the average variation in the injections angles for the two flux surfaces of all three scenarios. The definitions and numbers are based on estimates from ITER PID [1]. The maximum number of full and partial rotations required during the lifetime of ITER is estimated based on confinement and sawtooth time scales, and on the burn cycles and pulse lengths. The maximum accumulated NTM rotational cycles are estimated as 21,000 full cycles and 840,000 partial cycles for the modulation rotations for the FS launcher at any angle within the mechanical working range of $\pm 6^{\circ}$. The desired steering accuracy has been determined by physics requirements to $\pm 0.05^{\circ}$. The full steering range is covered in less then $5 \mathrm{~s}$ for a period without loss of range.

\section{A. First steering mechanism prototype}

The first prototype, see Fig. 2, is being built for the purpose of demonstrating and validating the pneumatic actuator concept combined with the pressure control system. Issues regarding machinability, assembly and welding sequences and dynamic response are investigated. While equivalent in terms of functional capabilities, this first prototype does neither integrate the ITER grade materials nor a working cooling circuit.

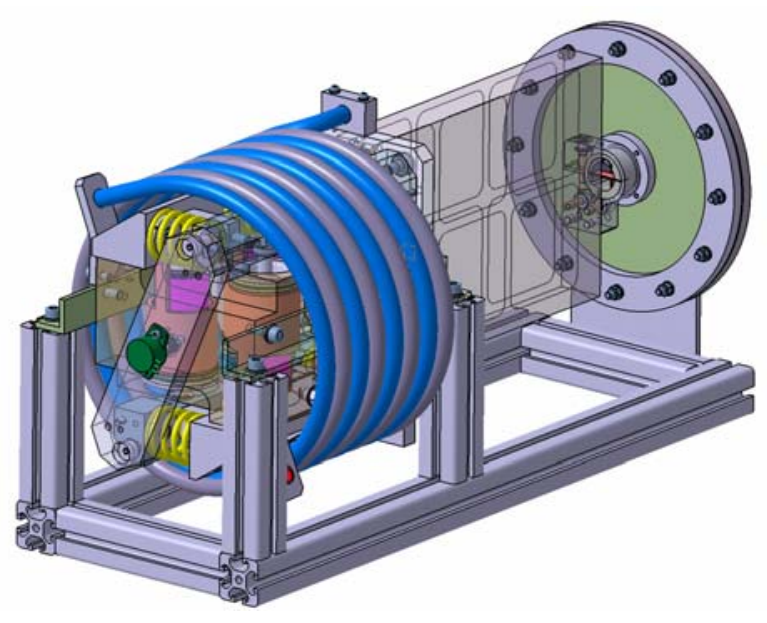

Figure 2. The first steering mechanism prototype

\section{B. Actuator}

The actuator, see Fig. 3 essentially consists of an externally pressurized bellows working against an elastically preloaded set of compressive springs. Achieving sufficient stiffness of the complete assembly while using bellows of adequate 
displacement capacity requires a working pressure range considerably above the squirming pressure limit (buckling failure due to elastic instability) of a bellows with internal pressurization. The selection of a bellows requires taking into account all relevant boundary conditions, in particular pressure, displacements and temperature gradient as a consequence of neutron irradiation. The currently retained manufacturing processes for the corrugated part of the bellows is a multilayer hydroformed system with a wide choice of available sheet materials, alternatively stainless steel and high nickel alloys. The likely material choice are laminates made of nickel base alloy such as Alloy 718 and 625. The relevant proprieties, in particular fatigue data after irradiation, are available in the ITER Material Proprieties Handbook [2]. Manufacturers generally refer to the standards of the Expansion Joint Manufacturers Association (EJMA code [3]). The cited conditions of applicability refer to bellows manufactured by a forming process, such as hydraulic expansion or rolling.

The rules on fatigue and life time estimation apply to austenitic steel or similar alloys only operating at less than $500^{\circ} \mathrm{C}$, a limit well above the value expected in the steering mechanism. The bellows is assimilated to a structure of general single-layer homogeneous type, without structural discontinuities within the working membrane part. In the case of a multi-layer hydroformed bellows this is not exactly true, but the relevant code [3] treats it as an equivalent single layer structure for stress and fatigue related calculations. The ends are welded to a supporting structure. The electron beam or laser welded joints are considered less critical than the working membrane part of the bellows and shall be analysed at a later design stage. The dpa rate (neutron damage as displacements per atom) was calculated to be $0.18 \mathrm{dpa}(0.355 \mathrm{dpa}$ full power year) for Alloy 718 at the location of the bellows, a value which is below the recommendation of 0.3 dpa fpy for the use of unirradiated physical properties, given in [2]. The effects of nuclear heating exist only for burning plasmas. The bellows are subject to volume heat loads due to neutron flux. At steering mechanism location, the volumetric heat influx into one bellows amounts to $\mathrm{dq} / \mathrm{dt}=0.8 \mathrm{MW} / \mathrm{m}^{3}$. For Alloy 718 , the specific heat is $\mathrm{cp}, \mathrm{C}=436 \mathrm{~J} / \mathrm{kgK}$, the mass density is $\rho=$ $80200 \mathrm{~kg} / \mathrm{m}^{3}$. The average rate at which the average temperature would rise in the bellows, made of Alloy 718, without heat lost through radiation or conduction, is $\mathrm{dT} / \mathrm{dt}=$ $\mathrm{dq} / \mathrm{dt} \bullet 1 /(\rho \bullet \mathrm{cp})=0.23 \mathrm{~K} / \mathrm{s}$. Without heat conduction or radiation, the temperature would increase by $230 \mathrm{~K}$ after 1'000 s.

The bellows are subject to and designed for the expected $21^{\prime} 000$ cycles over the full steering range from $-7^{\circ}$ to $+7^{\circ}$, and $840^{\prime} 000$ partial cycles at $\pm 2^{\circ}$ at any position within the steering range. The bellows are subjected to simultaneous axial, lateral and angular displacements, while being exposed to a variable external pressure which is a function of position, itself imposed by the elastic reaction of the preloaded compressive springs and cooling pipes.

The use of the precipation hardenable Alloy 718 requires a thermal treatment (precipitation hardening) after hydroforming of the bellows. Several treatments have been applied to manufactured bellows in order to determine a relationship linking the thermal history with cyclic fatigue and to verify the effects of the high rates of strain undergone during the hydroforming process of laminated strip metal. The optimized thermal process results in the bellows survival to at least 250'000 equivalent cycles (calculated with EJMA code [3]) over the full steering range.

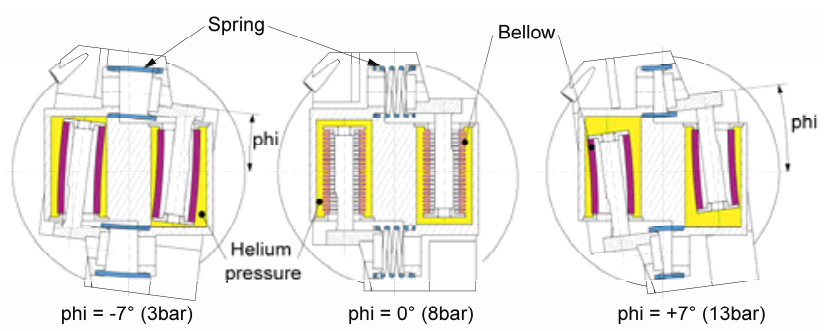

Figure 3. Radial cross section of the FS steering mechanism, through the axis of the bellows, shown in extension (left view), neutral (center), and compressed (right)

The purpose of the set of compressive springs is the elastic preloading of the steering mirror rotor against the fixed stator. The bellows work against the springs with a given force, resulting from the applied gas pressure and reduced by the force necessary to expand the bellows themselves. The force respectively moment balance includes elastic contributions from the flexure pivots and the set of helical cooling tubes. The signs of these contributions and their amplitude depends on how these components are fixed relative to their stressless state into the mechanism. Hence the springs have to equilibrate the sum of all forces and moments respectively for a given angular position. The deformation of the springs is proportional to the expansion of the bellows and equal if the radii to the axis of mirror rotation have identical value. The typical compressive spring will have to work over a stroke of $17 \mathrm{~mm}$, while having both ends rotated relative to each other by as much as $\pm 7^{\circ}$ relative to angular reference position. The spring force required ranges from $124 \mathrm{~N}$ in the most extended position, to $263 \mathrm{~N}$ in the most compressed position. The resulting spring stiffness rate is around $8 \mathrm{~N} / \mathrm{mm}$. The preferred spring type is the helical standard spring, made of wire with circular cross section. Alternatively a machined spring would offer the advantage of weldable ends.

\section{Bearings}

Cross flexure pivots (CFP), see Fig. 4, are backlash free and frictionless, and have excellent repeatability with low hysteresis, are self centering, maintenance free, vacuum compliant and do not require lubrication. CFPs are generally constructed utilizing flat elastic strips acting as flexure springs and which are locked and symmetrically crossed at a $90^{\circ}$ angle and encapsulated in a cylindrical housing. The primary requirements on the flexure pivot are the angular rotation and radial loading capabilities. Since the forces applied are symmetric relative to the rotations axis and internal to the steering mechanism, the rotation angle is imposed by pure moments along the axis of rotation. In absence of thermally and electromagnetically induced structural loads, the stresses arising within the flexure pivot are the result of forced rotations only. Since the beam is deflected at twice the mirror steering angle, the flexure pivot is required to rotate $\pm 7^{\circ}$ (mechanical margins included). The flexure pivot is fixed in the steering 
mirror assembly in accordance with the centered reference angle, which is likely to coincide with the neutral angle of the flexure pivot. Rotation angles larger than the design angle are prohibited and made impossible by design, with mechanical end stops built into the rotor/stator contact faces.
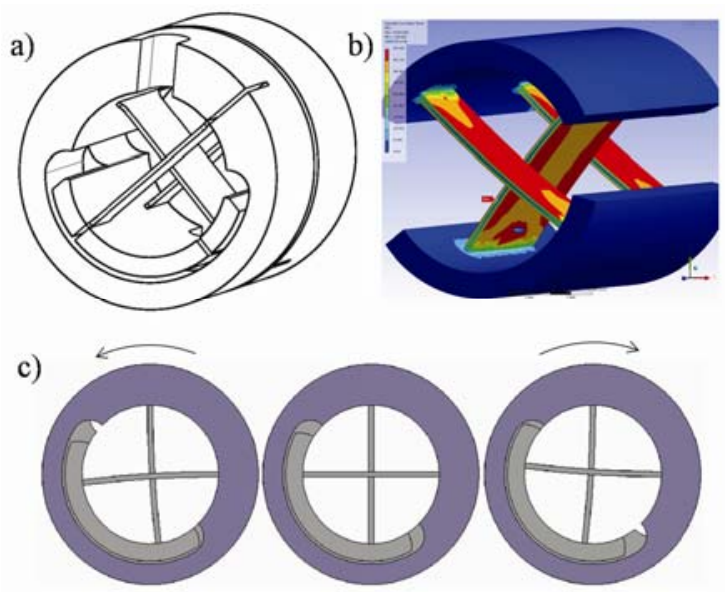

Figure 4. a) Flexure pivot in Titanium, b) Equivalent Von Mises Stress for a $7^{\circ}$ rotation, $\max 497 \mathrm{MPa}, \mathrm{c}$ ) working principle.

The flexure pivot centers the rotor without radial and axial clearance. Because the working forces are mostly tangential and balanced within the cartridge, there is virtually no radial or excentric load on the rotor other than electromagnetically induced forces.

The EM forces [4] related to the currents induced in the rotating mirror during a plasma disruption event were estimated for the steering mirror in the worst configuration, assuming no shielding effect from the port wall, $\mathrm{dB}_{\mathrm{P}} / \mathrm{dt}=25 \mathrm{~T} / \mathrm{s}$ and $\mathrm{B}_{\mathrm{T}}=5$ T. The mirror dimensions are $230 \mathrm{~mm}$ (toroidal), $196 \mathrm{~mm}$ (poloidal) and $30 \mathrm{~mm}$ (thickness), with the reflecting conductive layer of $2 \mathrm{~mm}$ made from copper. The resulting net torque is $1 \mathrm{kNm}$ on the mirror rotor. (a flexure pivot is positioned on each side of steering mirror). Du to the flexure pivot arrangement, the radial loads are $6400 \mathrm{~N}$ for the balanced system. The flexure pivots are capable of supporting up to 6700 N.

\section{STEERING MECHANISM SERVOCONTROL}

The pressurized bellows are necessary to overcome the combined stiffness of the cooling coils, the pivots and the springs. To cover the full angular movement, pressure is modulated between 0.3 and $1.3 \mathrm{MPa}$, which proves to be sufficient to control the mirror in stable conditions. Helium as a pressure transmitter is a good choice not only for its chemical and nuclear properties, but additionally for the high acoustic velocity. The acoustic velocity marks the limit of speed for a pressure signal traveling through the pipe and thus determines the pipe delay time and the overall dynamic range of the system.

The radiation and temperature sensitive pressure control servovalves are located in the port duct behind the closure plate, where environmental conditions are acceptable.
The typical mobile mirror system is composed of an electronic amplifier or discrete controller adjusting the solenoid current proportional to the error on pressure driving the servovalve, which releases a certain amount of gas into the bellows piston. The piston acts against the preloaded mirror, until elastic displacement and pressure at the piston are equalized.

Although the mirror angular equilibrium position is determined by the pneumatic actuator pressure balancing the elastic moments, essentially provided by the compressive springs, the addition of a real time in-situ angular sensing device may be desirable for improving the dynamic response of the system. Various sensor principles are currently under evaluation.

\section{CONCLUSION}

All $\mathrm{q}=2 \& 3 / 2$ surfaces envisioned can be accessed with a FS launcher, which has above marginal performance for stabilizing the NTMs. A preliminary prototype design of the steering mechanism has been made, which avoids all frictional surfaces and all induced stresses are within ITER limits. Future plans include developing a more detailed engineering design leading to construction of a second prototype launcher for testing. The FS launcher has been designed with the aim to improve the operating reliability and increase the physics capabilities of the upper port ECH launcher. Particular attention has been given to the design of a frictionless and backlash-free steering mechanism, which offers improved reliability and steering precision than that offered in present day FS launchers. Cyclic fatigue limits are within ITER lifetime requirements. Future plans include the extensive mockup testing to demonstrate the expected fatigue limits and the optimisation of the dynamic behaviour of the mirror rotor.

\section{ACKNOWLEDGMENT}

This work was supported by the Swiss National Science Foundation. This work, supported by the European Communities under the contract of Association between EURATOM/ CRPP-EPFL, was carried out within the framework of the European Fusion Development Agreement. The views and opinions expressed herein do not necessarily reflect those of the European Commission.

\section{REFERENCES}

[1] J. How, ITER Project Integration Document, ITER Design Integration Division, Release 3.0 (2007).

[2] ITER Material Proprieties Handbook (MPH)

[3] EJMA code - Standards of the Expansion Joint Manufacturers Association Inc., 8th ed., $2003+2005$ Addenda +2006 Errata. www.ejma.org.

[4] M.A. Henderson, "Design Status of the ITER Upper Port Launcher", unpublished, IAEA ITER ECH meeting in Vienna

[5] F. Sanchez et al. "Design status of ITER ECH upper launcher mirrors", this conference, $P 3-8$

[6] J.-D. Landis et al. "Design of the critical components in the ITER ECH upper launcher steering mechanism", unpublished, accepted for publication in Fusion Engineering Design (2007), Elsevier. 